\title{
A TD-DFT Treatment of Electronic Excitations in the STEM Spanning Dipole and Impact Scattering Regimes
}

\author{
David Lingerfelt ${ }^{1 *}$, Jacek Jakowski ${ }^{1,2}$, Panchapakesan Ganesh ${ }^{1}$, and Bobby Sumpter ${ }^{1}$ \\ 1. Center for Nanophase Materials Sciences, Oak Ridge National Laboratory, Oak Ridge, USA. \\ 2. Computational Sciences and Engineering Division, Oak Ridge National Laboratory, Oak Ridge, USA. \\ * Corresponding author: LingerfeltDB@ornl.gov
}

The potential for lasers to drive chemical reactions by delivering electric fields comparable in strength to the internal fields in molecules was realized not long after their invention. [1] With the advent of ultrafast optical pulse shaping methods [2] and theoretical developments in the areas of quantum optimal control [3] and electronic structure theory, a pathway towards individual bond photochemistry was cleared. Along a parallel path toward chemical manipulations with individual bond/atom specificity, monochromated and aberration corrected STEM instrumentation (developed to enable picometer resolution imaging) has been re-envisioned as a tool for modifying materials at the atomic scale [4]. In addition to emanating inherently localized (point source) electric fields, beam electrons carry significant linear momentum that can be imparted to nuclei through elastic scattering, positioning convergent electron beam irradiation as an appealing option for initiating nonequilibrium electronic and vibrational dynamics in materials with atomic spatial resolution. Unfortunately, a currently unmet need for software capable of modeling the electronically non-adiabatic response of materials to the impact of swift charged particles has stifled progress in the development of atomic-scale manipulation and fabrication methods in the STEM.

In this paper, we describe our adaptation of an inexpensive-yet-accurate excited state electronic structure method to treat neutral electronic excitations of materials promoted by interactions with external charged particles. While the electronic excitation of materials by swift electrons has previously been approached as an inelastic scattering problem for the incoming electron [5], we invert this perspective and instead consider the electronic response of the material subjected to perturbation by an external point charge using time-dependent electronic structure theory.

Linear response time-dependent density functional theory (TD-DFT) [6] provides a formalism to solve for the excited electronic states of an isolated material and calculate the elements of the potential matrix which couple the ground and excited electronic states through the presence of an external point charge. These coupling matrix elements were evaluated in a locally modified version of the NWchem electronic structure code $[7,8]$, and used (through the application of first-order time dependent perturbation theory) to determine the position-dependent, point charge induced transition rates between electronic states. Ground to excited state transition rates were calculated for a simple atomic system due to point charge perturbations across a range of separation distances and validated against the excited state populations from time-domain TD-DFT simulations of the material's electronic response to point charge impulses.

It is well known that the commonly-employed electric dipole approximation to the interaction between the material's electrons and the evanescent field of a beam electron is only strictly valid in the limit of zero momentum transfer from beam to material [5]. We present a simple method (relying on our ab initio treatment of the matter-beam interaction) to determine when dipole selection rules fail to accurately predict electronic excitations induced by the presence of a nearby charged particle. Finally, the role of electronic non-adiabaticity in facilitating an exemplary structural transformation of a substitutionally 
doped polybenzoid is examined. The methodologies described here will contribute to a more complete understanding of the dynamics induced in materials under irradiation by electron beams as they manifest in cathodoluminescence, electron beam induced current, and structural transformations of materials.

\section{References:}

[1] D. W. Lupo, M. Quack, Chemical Reviews 87 (1987), p. 181.

[2] A. M. Weiner, Progress in Quantum Electronics, 19 (1995), p. 161.

[3] A. P. Peirce, M. A Dahleh, H. Rabitz, Physical Review A, 37 (1988), p. 4950.

[4] S. V. Kalinin, A. Borisevich, S. Jesse, Nature News, 539 (2016), p. 485.

[5] M. Inokuti, Reviews of Modern Physics, 43 (1971), p. 297.

[6] A. Dreuw, M. Head-Gordon. Chemical Reviews, 105 (2005), p. 4009.

[7] M. Valiev et al., Computer Physics Communications, 181 (2010), p. 1477.

[8] D. B. Lingerfelt et al., ChemRxiv, (2019), 7726139
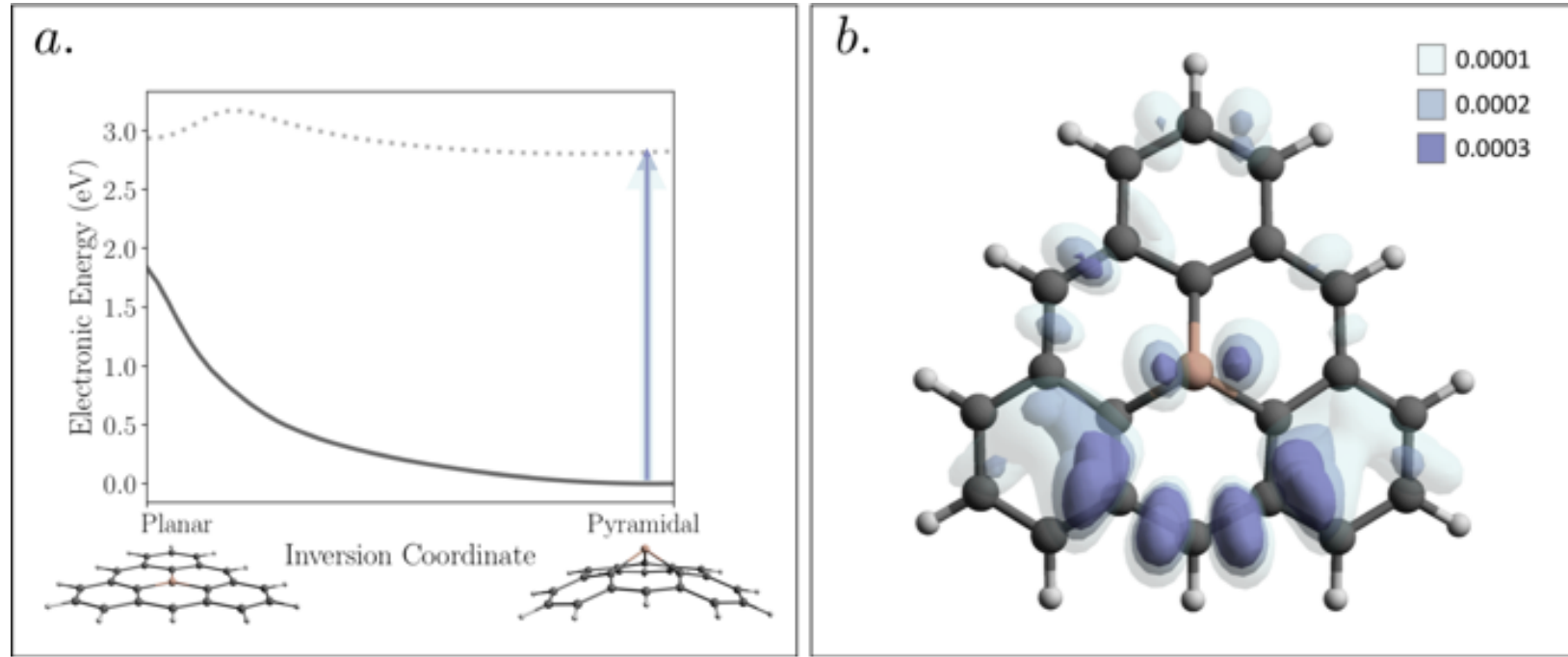

Figure 1. (a.) Potential energy surfaces along the ground-state minimum energy pathway for inversion of a small phosphorous-doped polybenzoid. Note that only half of these symmetric reaction profiles are plotted, ranging from the optimized equilibrium geometry (pyramidal) to that of the inversion transition state (planar). Dramatic reduction in the barrier for inversion is observed in the first excited state. The position-dependence of the rate of transition to this state induced by a particle of elementary charge is visualized $(b$.) as isosurfaces (rates per atomic time unit indicated by color), demonstrating the potential for an electron beam to simultaneously promote electronic excitation to an inversion-favoring state (inelastic scattering contribution) and transfer some linear momentum to the defect site (elastic scattering contribution) to initiate the inversion. These early results allude to a nontrivial role of inelastic scattering and electronically non-adiabatic vibrational dynamics in facilitating some structural modifications that occur in the (S)TEM. 